\title{
Prognostic Factors and Tumor Infiltrating Lymphocytes in Triple Negative Breast Cancer
}

\author{
Özgecan Dülgar ${ }^{1}$ (D), Şennur İlvan² (1), Zeynep Hande Turna ${ }^{3}$ (1) \\ ${ }^{1}$ Departmant of Medical Oncology, İstanbul Medeniyet University Göztepe Training and Research Hospital, İstanbul, Turkey \\ ${ }^{2}$ Deptartment of Pathology, İstanbul University-Cerrahpaşa, Cerrahpasa School of Medicine, İstanbul, Turkey \\ ${ }^{3}$ Department of Medical Oncology, İstanbul University-Cerrahpaşa, Cerrahpasa School of Medicine, İstanbul, Turkey
}

\section{ABSTRACT}

Objective: Triple-negative-breast-cancer (TNBC) is a very heterogenous disease some of which are very aggressive and have poor prognosis. No targeted therapy is available. Immune response and tumor-infiltrating lymphocytes (TIL) can be related to longer disease-free survival (DFS) and overall survival (OS) in TNBC. Family history of cancer can be related poor prognosis, irrespective of genetic mutation.

Materials and Methods: Pathology reports and files of 167 patients operated for TNBC were assessed retrospectively. The effects of lymphocyte infiltration, family history of cancer and other tumor characteristics on prognosis were evaluated. Data of 137 patients was included in statistical analysis.

Results: Univariate-analysis revealed that stage, size of tumor, histological subtype, number of infiltrated axillary lymph-nodes, lymphatic and vascular invasion, choice of adjuvant/neoadjuvant chemotherapy, family history of cancer has a statistically significant effect on DFS. Increase in density of lymphocyte infiltration of tumor has also better a prognostic effect on DFS ( $\mathrm{p}=0.02)$. In multivariate-analysis, only tumor size and choice of adjuvant/neoadjuvant chemotherapy are found to have statistically significant effect.

Conclusion: Tumor lymphocyte infiltration was found to have a statistically significant better prognostic effect on DFS but not on OS of patients with operated TNBC. This result can be due to variability of therapies administered after recurrence and other confounding factors that may have an effect on OS.

Keywords: Family history, prognosis, triple negative breast cancer, tumor lymphocyte infiltration

Cite this articles as: Dülgar Ö, İlvan Ş, Turna ZH. Prognostic Factors and Tumor Infiltrating Lymphocytes in Triple Negative Breast Cancer. Eur J Breast Health 2020; 16(4): 276-281.

\section{Introduction}

Triple negative breast cancer (TNBC) lacking estrogen receptor (ER), progesterone receptor (PR) and human epidermal growth factor receptor-2 (HER-2) is a very heterogeneous disease some of which are very aggressive and have poor prognosis. Although racial differences are seen, TNBC consists 10-15\% of all breast cancers (1) and are more prevalent among premenopausal women with BRCA-1 mutation (2).

Patients with TNBC may have shorter disease-free-survival (DFS) and overall-survival (OS). No targeted therapy is available (2). Seventy five percent of TNBC is basaloid subtype with which has a shorter DFS (3). In a study involving 1601 breast cancer patients for comparing TNBC with other types of breast cancer, TNBC was diagnosed at younger ages and the tumors had a larger tumor sizes and higher grades with higher metastatic capacity (3).

Immune response plays an important role in cancer development and controlling tumor progression Tumor-infiltrating lymphocytes (TIL) reflect the local immune response. Tumor lymphocyte infiltration has been demonstrated in melanoma, colorectal carcinoma, renal cell carcinoma and lung cancer with a positive effect on patient survival. New therapies such as immune system checkpoint inhibitors have come into clinical practice in treatment of cancer types (4).

In some publications, tumor lymphocyte infiltration in breast cancer has no effect on prognosis (5) or is associated with poor prognosis (6), although in other studies, it is considered to be a good prognostic marker $(7,8)$. TIL is associated with a better prognosis in fast grow- 
ing types of breast cancer and has also been associated with longer DFS and OS in TNBC and may have effect on better treatment response in HER-2 positive tumors (9). Breast cancer is a heterogeneous disease and TIL have different immunophenotypic characteristics, and the pathological evaluation of lymphocyte infiltration is not standardized. These could be the reasons of discrepancies in previous studies.

\section{Materials and Methods}

\section{Patients}

The files of patients with TNBC admitting to Medical Oncology Department between January 2004-December 2014 were retrospectively analyzed. The files of 30 patients were excluded because of missing data and a total of 137 patients with operable TNBC patients were enrolled in the study. The following patients without surgery were excluded from the study; 8 patients who progressed after neoadjuvant chemotherapy, 2 patients were diagnosed with carcinoma in situ, 2 patients were not operated because of advanced age, and 7 patients' data was insufficient for analysis. Patients provided written informed consent. The study was approved on November $24^{\text {th }}$, 2015, Ethics Committee of Istanbul University-Cerrahpasa, Cerrahpaşa School of Medicine with number of $83045809 / 604.01$.

\section{Definition of clinic parameters}

Tumor and patient characteristics were evaluated retrospectively from the file registry. The TNM staging was determined according to the seventh edition of the American Joint Committee on Cancer. Overall survival and DFS times were defined as the time from operation to death and to recurrence of breast cancer, respectively (10).

\section{Definition of pathologic parameters}

Hormone receptor status was defined as positive when immunohistochemistry test results for ER PR were positive and defined as negative when both tests results were negative. HER2 expression was defined as negative when the immunohistochemistry results were negative or $1+$ and defined as positive when the results were $3+$. When the results were $2+$, we defined the negativity of HER2 according to the results of the fluorescence in situ hybridization test. The American Society of Clinical Oncology/College of American Pathologists guideline recommendations for immunohistochemical testing of ER, PR, and HER2 were used $(11,12)$. Surgical specimens were evaluated by two different pathologists. The ratio of stromal tumor-infiltrating lymphocyte (sTIL) was measured in primary tumor specimen with using visual assessment of standard hematoxylin and eosin (H\&E) stained sections. TIL was classified in four groups as none, low, moderate and high. A point of 0 indicated none of sTIL; $1+$, low sTIL $(<30 \%) ; 2+$, moderate

\section{Key Points}

- $\quad$ TNBC is heterogenous disease with different prognosis. We evaluated prognostic role of TIL, family history of cancer and classical prognostic factor. In our study including women with operable TNBC who had long follow-up, Increased TIL rate associated good prognosis. High TIL rate may be predicting response to immunotherapy. Further prospective trials to prove the predictive effect of TIL are necessary.

- Women who have breast cancer related genetic mutation (e.g. BRCA1-2) often have a family history of cancer but small group of breast cancers are thought to be hereditary and can be using targeted therapy.

- Family history of cancer can be prognostic effect with or without genetic mutation.
(30\%-60\%); and 3+, marked high lymphocytic infiltration (>60\%). We used semiquantitative approaches like some other studies (13).

\section{Statistical analysis}

Data of study was recorded and analyzed using Microsoft Excel' 2010 (Microsoft Corporation, Redmond, WA) IBM Statistical Package for the Social Science version 20.0 (IBM SPSS Corp., Armonk, NY, USA). The Kaplan-Meier method was used for estimation of disease free and overall survival rates, and the log-rank test was used to determine the significance of differences between two or more survival curves. The Cox proportional hazards model was used for univariate and multivariate analysis, and the hazard ratio was calculated according to the cutoff value of a $95 \%$ confidence interval (CI). All tests were two-sided and we regarded the results of statistical analyses as significant when the $p$-value was less than 0.05 .

\section{Results}

\section{Patient characteristics}

Patients median age was $52.9 \pm 12.7 .112$ of patients were postmenopausal and 55 of patient was premenopausal at the time of diagnosis. 94 patients have history of cancer at least in one family member.

\section{Pathological characteristics}

A total of 8 different histologic subtypes were detected, the majority of the patients have invasive ductal carcinoma. Most of the tumors were grade 3 and there were no patients with grade 1 tumor. The TNM stage and pathological characteristics of tumors were summarized in Table 1.

\section{Results of DFS and OS}

\section{Prognostic impact of tumor infiltrating lymphocytes}

Increasing TILs were associated longer DFS. Rate of TILs were evaluated in four groups as none, low, moderate and high. DFS was $22.5 \pm 7.5$ months, $83.8 \pm 8.6$ months, $90.8 \pm 7.8$ months and $118.8 \pm 10.9$ months, respectively. TIL in tumor tissue was found to have a significant prognostic effect on DFS of patients with TNBC ( $\mathrm{p}=0.022)$. Prognostic impact of tumor infiltrating lymphocytes on DFS can be seen in Figure 1. In patients with tumors who have high TILs, DFS was significantly longer, whereas there was no statistical significance in terms of OS $(\mathrm{p}=0.78)$.

\section{Prognostic impact of stromal desmoplasia and tumor infiltrating plasmocytes}

Patients with tumors without stromal desmoplasia DFS was $27 \pm 7$ months whereas $71.8 \pm 12$ months in patients with tumors with high stromal desmoplasia. This difference was not found to be statistically significant $(\mathrm{p}=0.607)$. Tumor infiltrating plasmocytes (TIP) were evaluated in four groups (none, low, moderate, high). DFS was $28 \pm 7.8$ months in patients with none TIP group while it was $117.5 \pm 11.4$ months in patients with high TIP group. On the other hand, this difference was not statistically significant $(\mathrm{p}=0.16)$.

\section{Prognostic impact of family history of cancer}

Median DFS time was 52 months in patients with family history of cancer and 133 months in patient without family history. The difference was statistically significant $(\mathrm{p}=0.03)$. Prognostic impact of family history of cancer on DFS can be seen in Figure 2. Triple negative breast cancer patients with family history have poor prognosis, irrespective of genetic mutation. 


\section{Prognostic factors and survival analysis}

Effect of degree of stromal lymphocyte and plasmocyte infiltration, desmoplasia (as in reports: none, low, moderate, and high) and classical prognostic factors on prognosis of early stage TNBC patients was investigated.

Univariate-analysis revealed that stage and size of tumor, lymphatic and vascular invasion has a statistically significant effect on both DFS and OS, however histological subtype, number of infiltrated axillary

Table 1. Pathological and clinical characteristics of the patients

\section{Pathological features of tumors}

Stages of patients

IA

IIA

IIB

IIIA

IIIB

IIIC

Histological subtypes

Invasive ductal carcinoma

Invasive lobular carcinoma

Mixt (ductal + lobular) carcinoma

Low differentiated carcinoma

Squamous carcinoma

Carcinosarcoma

Neuroendocrine tumor

Grade

2

3

Lymphatic invasion

Present

Absent

Vascular invasion

Present

Absent

Perineural invasion

Present

Absent

Number

Multicentricity

Present

Absent

Multifocal

Present

Absent
137

18

55

27

21

3

13

122

5

5

2

1

1

1

129

19

110

130

63

67

128

18

113

130

22

108

$\%$ 100

13.1

40.1

19.7

15.3

2.1

9.5

89.1

3.6

3.6

1.5

0.7

0.7

0.7

14.7

85.3

48.5

51.5

13.7

86.3

16.9

83.1

133

6

127

4.5

95.5

134

16

11.9

118 lymph-nodes, choice of adjuvant/neoadjuvant chemotherapy and family history of cancer has statistically significant effect only on DFS. Increase in density of lymphocytic infiltration of tumor was found to have a statistically significant effect on DFS $(p=0.02)$. The effects of prognostic factors on DFS and OS in our study are summarized in Table 2.

\section{Discussion and Conclusion}

TNBC is a heterogenous disease with different prognosis and have no specific targeted therapy $(14,15)$. In this trial we aimed to investigate the prognostic effect of tumor characteristics, and stromal immune response to tumor in patients with TNBC.

In our trial median DFS and OS of operable TNBC patients were found to be 91 and 168 months respectively. In a trial in Toronto, patients with TNBC were compared with other types of breast cancer patients. The mean DFS was 2.6 years and mean OS was 4.2 years (3). TNBC had the highest likelihood of distant recurrence in the first 2 years $(3,15)$. In our trial, 42 patients had recurrence: 28 patients $(67 \%)$ in the first 2 years and $38(91 \%)$ in 5 years. Few patients with

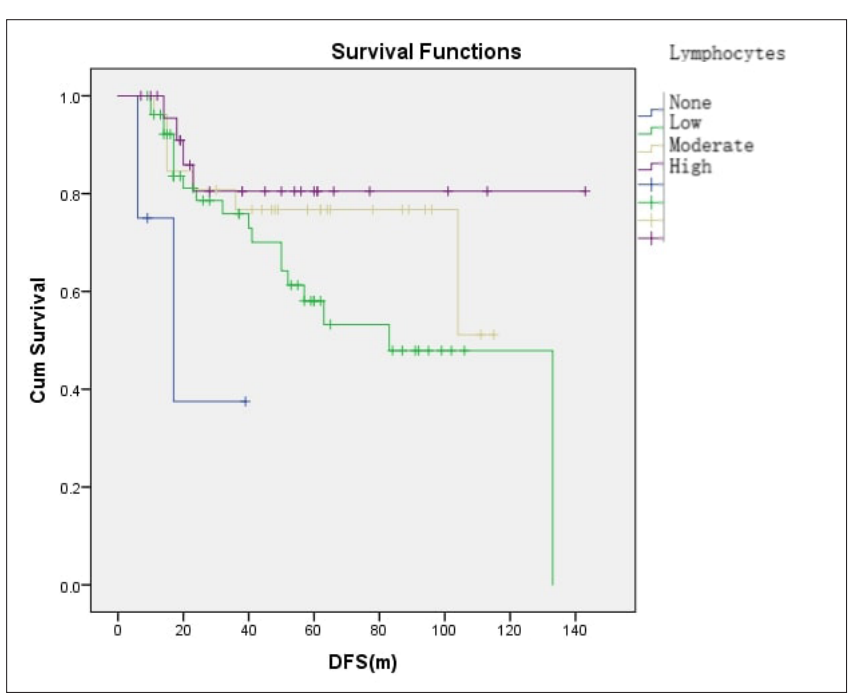

Figure 1. Prognostic impact of tumor infiltrating lymphocytes on DFS DFS: disease free survival

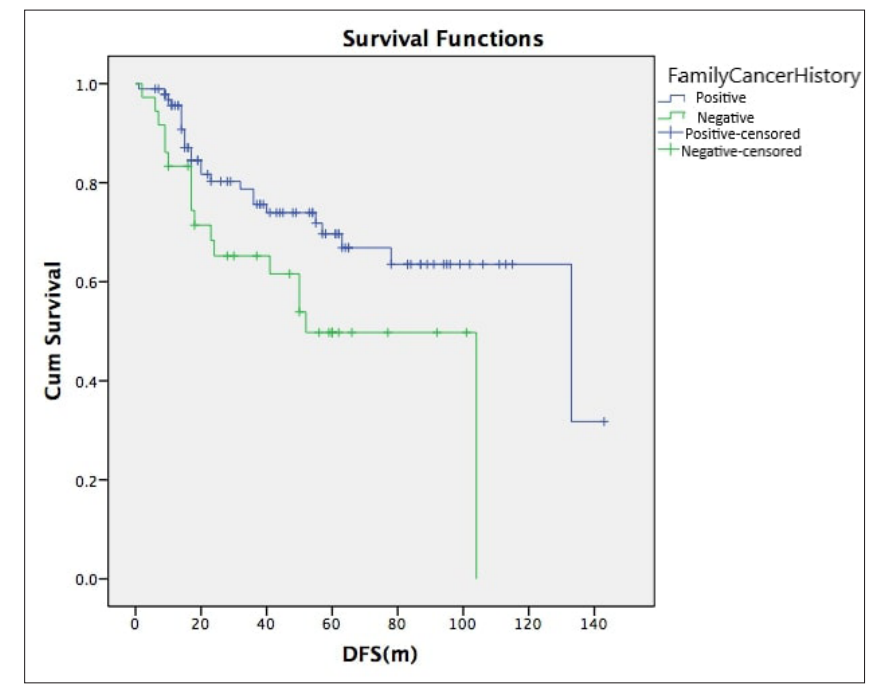

Figure 2. Prognostic impact of family history of cancer on DFS DFS: disease free survival 
Table 2. The effects of prognostic factors on DFS and OS in univariate and multivariate cox regression analyses

\begin{tabular}{|c|c|c|c|c|}
\hline $\begin{array}{l}\text { Prognostic } \\
\text { factors }\end{array}$ & $\begin{array}{c}\text { DFS } \\
\text { (univariate } \\
\text { analysis) } \\
\text { (p value) }\end{array}$ & $\begin{array}{c}\text { DFS } \\
\text { (multivariate } \\
\text { analysis) } \\
\text { (p value) }\end{array}$ & $\begin{array}{c}\text { OS } \\
\text { (univariate } \\
\text { analysis) } \\
\text { (p value) }\end{array}$ & $\begin{array}{c}\text { OS } \\
\text { (multivariate } \\
\text { analysis) } \\
\text { (p value) }\end{array}$ \\
\hline Stage & $<0.001^{*}$ & 0.062 & $0.008^{*}$ & 0.197 \\
\hline Tumor-size (T stage) & $0.001 *$ & 0.353 & $0.005^{*}$ & 0.019 \\
\hline Family History of Cancer & $0.003^{*}$ & & 0.006 & \\
\hline Lymphatic invasion & $0.012^{*}$ & 0.790 & 0.091 & 0.197 \\
\hline Vascular invasion & $0.005^{*}$ & 0.447 & $0.023^{*}$ & 0.229 \\
\hline Perineural invasion & 0.533 & & 0.620 & \\
\hline Numbers of Involved Axillary Lymph Nodes & $0.001 *$ & 0.878 & 0.26 & 0.827 \\
\hline Tumor-Infiltrating Lymphocyte & $0.022^{*}$ & 0.931 & 0.638 & 0.976 \\
\hline Tumor-Infiltrating Plasmocyte & 0.160 & & 0.61 & \\
\hline Stromal Desmoplasia & 0.607 & & 0.94 & \\
\hline Adjuvant/neoadjuvant Chemotherapy & $<0.001 *$ & 0.007 & 0.189 & 0.151 \\
\hline Histological Subtype & $0.035^{*}$ & 0.923 & 0.76 & 0.591 \\
\hline
\end{tabular}

TNBC had no recurrence after 10 years follow up showing the heterogeneity of disease.

Some publications report that TLI does not have a significant effect on breast cancer prognosis (5) or is associated with poor prognosis (6), while TLI is determined to be a good prognostic marker in some other studies $(7,8)$. In a study 47 ductal carcinoma tissues were analyzed by flow cytometry and $\mathrm{T}$ lymphocyte infiltration was predominantly CD8+ T cells followed by CD4 + T lymphocytes and CD56 + NK cells (16). In two different studies evaluating CD8 + T cells and lymphocyte infiltration intensity was shown to be directly related to young age, high grade, medullary histology, estrogen receptor negativity, basal subtype and better overall survival $(7,8)$. In one of these studies lymphocyte infiltration intensity was correlated with HER-2 negativity (7) and in the other with HER-2 positivity (8). The favorable prognostic effect on OS was confirmed only in the basaloid subtype and not detected in other triple negative tumors $(7,8)$.

TIL has been shown to be a good prognostic factor in tumors with high proliferation rates, such as triple negative and HER-2 + tumors $(9,17)$. No significant prognostic effect was seen in HER-2 negative (luminal A and B) and HER-2 and ER + tumors (17). In studies of TNBC evaluating intratumoral and stromal lymphocyte infiltration, each $10 \%$ increase in TIL was associated with reduced distant metastasis and mortality rate $(9,17,18)$. The group with lymphocyte infiltration more than $50 \%$ of the stroma has the best prognosis in both triple negative and HER-2 + tumors $(9,17)$.

In our study TIL was determined in four different groups: none, low, moderate and high. and mean DFS was 23 months for the patients without lymphocyte infiltration and was 119 months for patients with high lymphocyte infiltration. This difference was statistically significant $(\mathrm{p}=0.022)$.
The effect of TIL scores on OS wasn't statistically significant ( $\mathrm{p}=0.638$ ). In a study, TNBC was separated into different two groups core basal and null type (5 markers negative), TIL scores effected only core basal subtype prognosis. There was no difference in OS of whole TNBC cohort and null type with high TIL score (8). We could not evaluate TNBC's subtypes and different treatment regimens used after recurrence may have also a confounding effect on OS. In our study, high TIL scores were found to have significant effects only on DFS but not OS.

In a study CD68+ macrophages infiltration is found to be higher in hormone receptor-negative tumors (19). In another study, CD4+ T lymphocytes played major role in a complete response to neoadjuvant chemotherapy. If post-chemotherapy residual tumor had high CD3, CD68 It was found to be associated with poor DFS (20). Plasmocytic infiltration occurs as a response to tumoral antigen (21). In our trial, plasmocyte infiltration was also evaluated and was associated with longer DFS and OS. However, this effect was not statistically significant.

We analyzed the survival effect of the desmoplasia density of the tumor stroma. Despite the high desmoplasia level was associated with longer DFS, this was not statistically significant $(\mathrm{p}=0.607)$. Desmoplasia had no significant effect on OS ( $\mathrm{p}=0.94)$. The importance of stromal desmoplasia is also unclear in the literature. In TNBC, adjacent, stroma of TNBC includes leukocyte activation, mononuclear leukocyte proliferation, interferon signaling pathways, hepatic fibrosis, T-helper cell differentiation and antigen presentation. Stromal reaction may have important implications for local recurrence (22).

In our study, stage, tumor size, lymphatic invasion, vascular invasion, number of axillary lymph nodes involved, histological subtype, adjuvant/neoadjuvant treatment, lymphocyte infiltration and classical prognostic factors were found to have significant effect on DFS. DFS of the patients who received adjuvant chemotherapy was sig- 
nificantly better than that of the patients who received neoadjuvant chemotherapy. This difference is disappeared on multivariate analysis. Neoadjuvant chemotherapy is used in patients with locally advanced breast cancer. So we think that the difference may be related to the advanced stage. Family history of cancer had also significant effect on DFS irrespective of genetic mutation. The recent trial shows that BRCA mutation carriers had significantly worse outcomes than non-carriers (23). Due to family history of cancer, young age of onset for breast cancer, bilateral breast cancer, triple negative breast cancer. We do not know our patient genetic mutations but family history of cancer can be a prognostic factor. Stage, tumor size, and vascular invasion also had a significant effect on OS. Tumor size was the only parameter that was significant in OS $(\mathrm{p}=0.019)$ in multivariate analysis.

The prognostic effect of lymphocyte infiltration in TNBC which we intend to focus on, was statistically significant in DFS analysis $(p=0.022)$. We could not show the same effect on OS. Some of the prognostic factors that are significant for DFS were not significant in the OS analyses This can be due to the different treatment applications after the recurrence confounding OS durations TIL has a prognostic effect in TNBC. Treatment options in TNBC are often limited to chemotherapy. Immunologic response may be effective in controlling the disease and strengthening this response with recently developed immunotherapy treatments may be considered promising for TNBC. Durable response with anti-PD1 or anti-PD-L1 was seen approximate $10 \%$ in unselected TNBC patients and improves only up to $20 \%-30 \%$ when patients are selected based on IHC-based PD-L1+ tumors. This low rate can be increased with using TIL to prediction (24).

The prognostic effect of lymphocyte infiltration in triple negative breast cancer, which we intend to focus on, was statistically significant in DFS analysis $(\mathrm{p}=0.022)$. Classical prognostic parameters, such as stage, size of tumor, lymphatic and vascular invasion, histological subtype, number of infiltrated axillary lymph-node, and choice of adjuvant/neoadjuvant chemotherapy has also statistically important effect on DFS. We could not show the effect of some parameters on the overall survival for the reasons we mentioned earlier. We think that lymphocyte infiltration around the tumor is a prognostic feature of triple negative breast cancer.

Ethics Committee Approval: Ethics committee approval was received for this study from the ethics committee of İstanbul University-Cerrahpaşa (No: 83045809/604.01).

Informed Consent: Written informed consent was obtained from patients who participated in this study.

Peer-review: Externally peer-reviewed.

Author Contributions: Concept - Ö.D.; Design - Ö.D.; Supervision Z.H.T.; Resources - Ö.D., Ş.İ.; Materials - Ö.D., Ş.İ.; Data Collection and/ or Processing - Ö.D., Ş.İ.; Analysis and/or Interpretation - Ö.D., Z.H.T.; Literature Search - Ö.D.; Writing Manuscript - Ö.D.; Critical Review - Ö.D., Z.H.T., Ş.İ.; Other -Ö.D.

Conflict of Interest: The authors have no conflicts of interest to declare.

Financial Disclosure: The authors declared that this study has received no financial support.

\section{References}

1. Pal S, Luchtenborg M, Davies EA, Jack RH. The treatment and survival of patients with triple negative breast cancer in a London population. Springerplus 2014; 3: 553. (PMID: 25324980) [CrossRef]

2. DeSantis CE, Fedewa SA, Goding Sauer A, Kramer JL, Smith RA, Jemal A. Breast cancer statistics, 2015: Convergence of incidence rates between black and white women. CA Cancer J Clin 2016; 66: 31-42. (PMID: 26513636) [CrossRef]

3. Dent R, Trudeau M, Pritchard KI, Hanna WM, Kahn HK, Sawka CA, Lickley LA, Rawlinson E, Sun P, Narod SA. Triple-negative breast cancer: clinical features and patterns of recurrence. Clin Cancer Res 2007; 13 : 4429-4434. (PMID: 17671126) [CrossRef]

4. Taube JM, Klein A, Brahmer JR, Xu H, Pan X, Kim JH, Chen L, Pardoll DM, Topalian SL, Anders RA Association of PD-1, PD-1 ligands, and other features of the tumor immune microenvironment with response to anti-PD-1 therapy. Clin Cancer Res 2014; 20: 5064-5074. (PMID: 24714771) [CrossRef]

5. Liburd EM, Pazderka V, Kovithavongs T, Dossetor JB. Evidence for suppressor cells and reduced CML induction by the donor in transplant patients. Transplant Proc 1978; 10: 557-561. (PMID: 152490)

6. Matkowski R, Gisterek I, Halon A, Lacko A, Szewczyk K, Staszek U, Pudelko M, Szynglarewicz B, Szelachowska J, Zolnierek A, Kornafel J. The prognostic role of tumor-infiltrating CD4 and CD8 T lymphocytes in breast cancer. Anticancer Res 2009; 29: 2445-2451. (PMID: 19596912)

7. Mahmoud SM, Paish EC, Powe DG, Macmillan RD, Grainge MJ, Lee AH, Ellis IO, Green AR. Tumor-infiltrating CD8+ lymphocytes predict clinical outcome in breast cancer. J Clin Oncol 2011; 29: 1949-1955. (PMID: 21483002) [CrossRef]

8. Liu S, Lachapelle J, Leung S, Gao D, Foulkes WD, Nielsen TO. CD8+ lymphocyte infiltration is an independent favorable prognostic indicator in basal-like breast cancer. Breast Cancer Res 2012; 14: R48. (PMID: 22420471) [CrossRef]

9. Loi S, Michiels S, Salgado R, Sirtaine N, Jose V, Fumagalli D, Kellokumpu-Lehtinen PL, Bono P, Kataja V, Desmedt C, Piccart MJ, Loibl S, Denkert C, Smyth MJ, Joensuu H, Sotiriou C. Tumor infiltrating lymphocytes are prognostic in triple negative breast cancer and predictive for trastuzumab benefit in early breast cancer: results from the FinHER trial. Ann Oncol 2014; 25: 1544-1550. (PMID: 24608200) [CrossRef]

10. Brody T. Clinical Trials Study Design, Endpoints and Biomarkers, Drug Safety, and FDA and ICH Guidelines. second ed 2016. 847 p.304-305.

11. Hammond ME, Hayes DF, Wolff AC, Mangu PB, Temin S. American society of clinical oncology/college of american pathologists guideline recommendations for immunohistochemical testing of estrogen and progesterone receptors in breast cancer. J Oncol Pract 2010; 6: 195-197. (PMID: 21037871) [CrossRef]

12. Wolff AC, Hammond ME, Hicks DG, Dowsett M, McShane LM, Allison KH, Allred DC, Bartlett JM, Bilous M, Fitzgibbons P, Hanna W, Jenkins RB, Mangu PB, Paik S, Perez EA, Press MF, Spears PA, Vance GH, Viale G, Hayes DF; American Society of Clinical Oncology; College of American Pathologists. Recommendations for human epidermal growth factor receptor 2 testing in breast cancer: American Society of Clinical Oncology/College of American Pathologists clinical practice guideline update. J Clin Oncol 2013; 31: 3997-4013. (PMID: 24101045) [CrossRef]

13. Schalper KA, Velcheti V, Carvajal D, Wimberly H, Brown J, Pusztai L, Rimm DL. In situ tumor PD-L1 mRNA expression is associated with increased TILs and better outcome in breast carcinomas. Clin Cancer Res 2014; 20: 2773-2782. (PMID: 24647569) [CrossRef]

14. Weigel MT, Dowsett M. Current and emerging biomarkers in breast cancer: prognosis and prediction. Endocr Relat Cancer 2010; 17: R245-262. (PMID: 20647302) [CrossRef]

15. Lin NU, Vanderplas A, Hughes ME, Theriault RL, Edge SB, Wong YN, Blayney DW, Niland JC, Winer EP, Weeks JC. Clinicopathologic features, patterns of recurrence, and survival among women with triple- 
negative breast cancer in the National Comprehensive Cancer Network. Cancer 2012; 118: 5463-5472. (PMID: 22544643) [CrossRef]

16. Leong PP, Mohammad R, Ibrahim N, Ithnin H, Abdullah M, Davis WC, Seow HF. Phenotyping of lymphocytes expressing regulatory and effector markers in infiltrating ductal carcinoma of the breast. Immunol Lett 2006; 102: 229-236. (PMID: 16246429) [CrossRef]

17. Loi S, Sirtaine N, Piette F, Salgado R, Viale G, Van Eenoo F, Rouas G, Francis P, Crown JJPA, Hitre E, de Azambuja E, Quinaux E, Di Leo A, Michiels S, Piccart MJ, Sotiriou C. Prognostic and predictive value of tumor-infiltrating lymphocytes in a phase III randomized adjuvant breast cancer trial in node-positive breast cancer comparing the addition of docetaxel to doxorubicin with doxorubicin-based chemotherapy: BIG 02-98. J Clin Oncol 2013; 31: 860-867. (PMID: 23341518) [CrossRef]

18. Adams S, Gray RJ, Demaria S, Goldstein L, Perez EA, Shulman LN, Martino S, Wang M, Jones VE, Saphner TJ, Wolff AC, Wood WC, Davidson NE, Sledge GW, Sparano JA, Badve SS. Prognostic value of tumor-infiltrating lymphocytes in triple-negative breast cancers from two phase III randomized adjuvant breast cancer trials: ECOG 2197 and ECOG 1199. J Clin Oncol 2014; 32: 2959-2966. (PMID: 25071121) [CrossRef]

19. Garcia-Martinez E, Gil GL, Benito AC, Gonzalez-Billalabeitia E, Conesa MA, García-Garre E, Vicente V, Ayala de la Peña F. Tumor-infiltrating immune cell profiles and their change after neoadjuvant chemotherapy predict response and prognosis of breast cancer. Breast Cancer Res 2014; 16: 488. (PMID: 25432519) [CrossRef]

20. Ruffell B, Au A, Rugo HS, Esserman LJ, Hwang ES, Coussens LM. Leukocyte composition of human breast cancer. Proc Natl Acad Sci U S A 2012; 109: 2796-2801. (PMID: 21825174) [CrossRef]

21. Wang Y, Ylera F, Boston M, Kang SG, Kutok JL, Klein-Szanto AJ, Junghans RP. Focused antibody response in plasma cell-infiltrated non-medullary (NOS) breast cancers. Breast Cancer Res Treat 2007; 104: 129-144. (PMID: 17393302) [CrossRef]

22. Casbas-Hernandez P, Sun X, Roman-Perez E, D’Arcy M, Sandhu R, Hishida A, McNaughton KK, Yang XR, Makowski L, Sherman ME, Figueroa JD, Troester MA. Tumor intrinsic subtype is reflected in cancer-adjacent tissue. Cancer Epidemiol Biomarkers Prev 2015; 24: 406-414. (PMID: 25465802) [CrossRef]

23. Wang YA, Jian JW, Hung CF, Peng HP, Yang CF, Cheng HS, Yang AS. Germline breast cancer susceptibility gene mutations and breast cancer outcomes. BMC Cancer 2018; 18: 315. (PMID: 29566657) [CrossRef]

24. Vikas P, Borcherding N, Zhang W. The clinical promise of immunotherapy in triple-negative breast cancer. Cancer Manag Res 2018; 10: 68236833. (PMID: 30573992) [CrossRef] 